\title{
Monoclonal Antibodies for Therapeutic Use: Specific Characteristics of Clinical Development, Evaluation by the Agencies, and Long-term Monitoring of Safety
}

\author{
Gilles Paintaud ${ }^{1}$, Marine Diviné ${ }^{2}$, Philippe Lechat ${ }^{3}$ and participants of Round Table N 5 of Giens XXVII* \\ 1 François Rabelais University Tours; CNRS UMR 7292; CHRU of Tours, Pharmacology-Toxicology Laboratory, Tours, France \\ 2 Amgen, France \\ 3 Afssaps, France
}

Received March 20th, 2012 ; accepted June $4^{\text {th }}, 2012$

\section{Keywords:}

monoclonal antibodies; clinical trials, phase I; clinical trials, phase II; clinical trials, phase IV

\begin{abstract}
Monoclonal antibodies (MoAb) are very different from other drugs. The Round Table aimed to determine whether the specific characteristics of MoAb have repercussions on their clinical development, evaluation by the health authorities, and long-term monitoring. As regards the structure-activity relationship of MoAb, classification according to mechanism of action (neutralising or agonist MoAb, cytolytic MoAb) is more relevant than to their degree of humanisation. Recommendations on their clinical development would be useful since the early phases give rise to a number of problems and are insufficiently codified. The pharmacokinetic profile is very different from that of other drugs. The concentration-effect relationship is difficult to study since the biomarkers may be apparently disconnected from the therapeutic effect. The methodology for evaluation of MoAb by the agencies, and postmarketing surveillance do not differ from the procedures used for other drugs; however, MoAb bring together a number of specific characteristics as compared with other drugs.
\end{abstract}

Abbreviations: see end of article.

\section{Introduction}

Monoclonal antibodies (MoAb) are playing an increasing role in treatment, and more than a hundred MoAb were in clinical development in 2011. These biopharmaceuticals (recombinant proteins produced by cells in culture) have a molecular mass and mechanisms of action which are very different from "conventional" drugs, generally obtained by chemical synthesis. The objective of the Round Table was to determine whether the specific characteristics of MoAb, in terms of structure and mode of action, have repercussions on their clinical development, evaluation by the health authorities, and long-term monitoring (safety, proper use and medical economics). Only "naked" MoAb, i.e. not conjugated with a radioactive isotope or toxin, and "whole" immunoglobulin $\mathrm{G}$ ( $\mathrm{IgG}$ ) have been discussed; the other forms of MoAb are special cases. Furthermore, biosimilars fall within a very different context and have not been considered. A previous Round Table on MoAb, which did not focus on their clinical development, was used as a basis for the discussions. ${ }^{[1]}$

\section{Structure-activity relationship}

The majority of therapeutic MoAbs currently on the market are IgG. These consist of two Fab portion ("ab" for antigen binding), able to bind to the antigen, and a Fc portion ("c" for crystallizable), responsible for the immunological and pharmacokinetic properties of MoAb (figure 1). IgG are made up of 2 heavy chains $(\mathrm{H})$ and two light chains (L). Variable domains (VH and VL) are found at the end of the Fab portions, each comprising 3 CDR (complementarity determining region) loops which, when combined (6 in total), represent the antigen epitope recognition site. The constant

\footnotetext{
* For the list of participants, see end of article.
} 


\section{CDR loops}

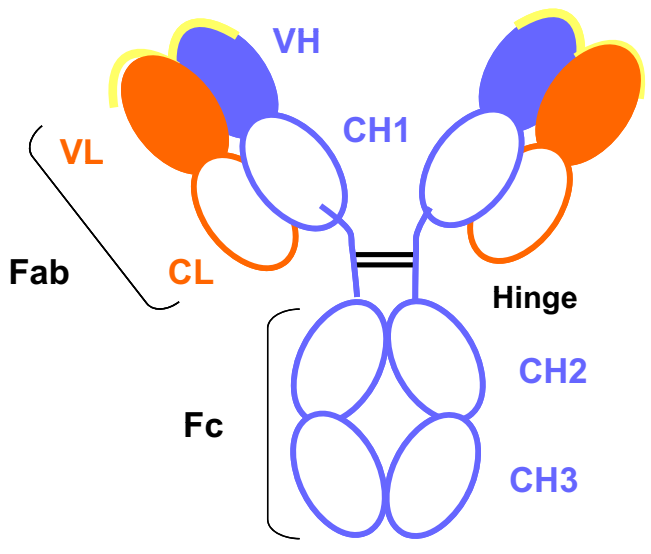

Fig. 1. Structure of immunoglobulin $G(\lg G)$. The variable parts are shown in colour. The Fab ("ab" for antigen binding) and Fc ("c" for crystallizable) portions and CDR (complementarity determining region) loops are shown.

portion (the sequence of which does not change within an Ig subclass in an individual) is made up of domains $\mathrm{CH} 1$ and $\mathrm{CL}$ (on the Fab portion) and domains $\mathrm{CH} 2$ and $\mathrm{CH} 3$ (on the Fc portion).

The first MoAbs were murine in nature, which resulted in a short half-life, low recruitment of immune effectors, and considerable immunogenicity. They were therefore gradually humanized, with the development of chimeric MoAb (suffix - ximab, in which only the variable portion is of murine origin), humanized MoAb (suffix -zumab, in which only the CDR are of murine origin), and finally fully human MoAb (suffix -mumab or -umab). ${ }^{[2]}$ However, from a clinical perspective, the distinction between chimeric, humanized and fully human MoAb may not be relevant since they all have a human Fc portion and also because certain fully human $\mathrm{MoAb}$ are proved to be immunogenic, such as adalimumab (Humira ${ }^{\circledR}$, an anti-TNF $\alpha$ ) in rheumatoid arthritis (see below). ${ }^{[3]}$

Classification of MoAb according to their mechanism of action is more relevant. They may act mainly via a neutralizing or agonist effect, or via a cytolytic effect, by recruiting immune effectors (figure 2). Neutralising MoAb bind to a soluble antigen, such as a cytokine, whose action they inhibit, as seen with bevacizumab (Avastin ${ }^{\circledR}$, an anti-vascular endothelial growth factor [antiVEGF]), or bind to a membrane receptor, which they block, as seen with MoAb directed against the epidermal factor receptor family (HER). For some MoAb, such as denosumab (an anti-receptor activator of nuclear factor-kappa B ligand antibody [anti-RANKligand]), the target antigen exists both in the circulating and membrane form. Binding may sometimes have an agonist effect if, when binding to the membrane receptor, they lead to post-receptor phenomena or apoptosis, as is the case for anti-tumor necrosis factor- $\alpha$ (TNF $\alpha)$ MoAb. ${ }^{[4]}$ As regards neutralising or agonist effects, the affinity of the MoAb for its target, hence its Fab portion, is the decisive factor for efficacy. These antagonist or agonist effects are not specific to MoAb as they may be achieved with fusion proteins or with small molecules, in contrast to the effects dependent of the Fc portion which are specific to the MoAb.

As regards cytolytic MoAb, the Fc portion also plays a very important role since it is responsible for recruiting immune effectors: complement $\mathrm{C} 1 \mathrm{q}$ fraction which leads to complementdependent cytotoxicity (CDC) or effector cells carrying the Fc $\gamma \mathrm{RI}-$ IIA/CD16 receptor, such as natural killer (NK) cells and macrophages, which lead to antibody-dependent cellular cytotoxicity (ADCC). ${ }^{[5,6]}$ A genetic polymorphism of FcyRIIIA influences its affinity for the antibody Fc portion. This polymorphism has been shown to be responsible for part of the variability in clinical response to cytolytic antibodies such as rituximab (MabThera ${ }^{\circledR}$, anti-CD20), ${ }^{[7]}$ trastuzumab (Herceptin ${ }^{\circledR}$, anti-HER2) ${ }^{[8]}$ and cetuximab (Erbitux ${ }^{\circledR}$, anti-EGFR). ${ }^{[9]}$

The Fc portion of MoAb may, moreover, be modified to suppress their affinity for Fc $\gamma \mathrm{R}$ receptors and, hence, their ADCC and CDC capacity, or, on the opposite, to increase their affinity and thus their cytolytic effects. These modifications concern the amino acid sequence or glycosylation of the Fc portion. ${ }^{[10,11]}$ When cytolytic MoAb are compared, particularly in cell models, it is therefore important to consider not only their affinity for the target antigen, but also to take into account the characteristics of their Fc portion. Certain in vitro potency tests are able to evaluate the quality of the Fc portion of a MoAb irrespective of its target antigen. The Fc portion of MoAb also has an impact on their pharmacokinetics (see below).

\section{Specific characteristics of clinical development}

\subsection{Diversity of the therapeutic areas, and medical economics}

In 2011, more than a hundred MoAb were in clinical development. Initially, the areas in which MoAb were successfully developed are oncology and rheumatology. Since 1999, 29 proprietary medicinal products have obtained marketing authorisation in 45 indications, and 21 proprietary medicinal products have been examined by the French National Authority for Health (Haute autorité de santé or HAS) with a view to reimbursement. The first $\mathrm{MoAb}$ (Orthoclone OKT3 ${ }^{\circledR}$, an anti-CD3), of murine origin, was on the market in 1986. The first fully human MoAb, adalimumab, was on the market in 2002, and 7 MoAb of this type existed in 2011. A large majority of MoAb on the market are indicated in oncology, lymphoproliferative disorders and in inflammatory disorders. However, the therapeutic areas for MoAb are expanding, into areas such as ophthalmology, autoimmune diseases (systemic lupus), cardiovascular system, infections, neurology (multiple 


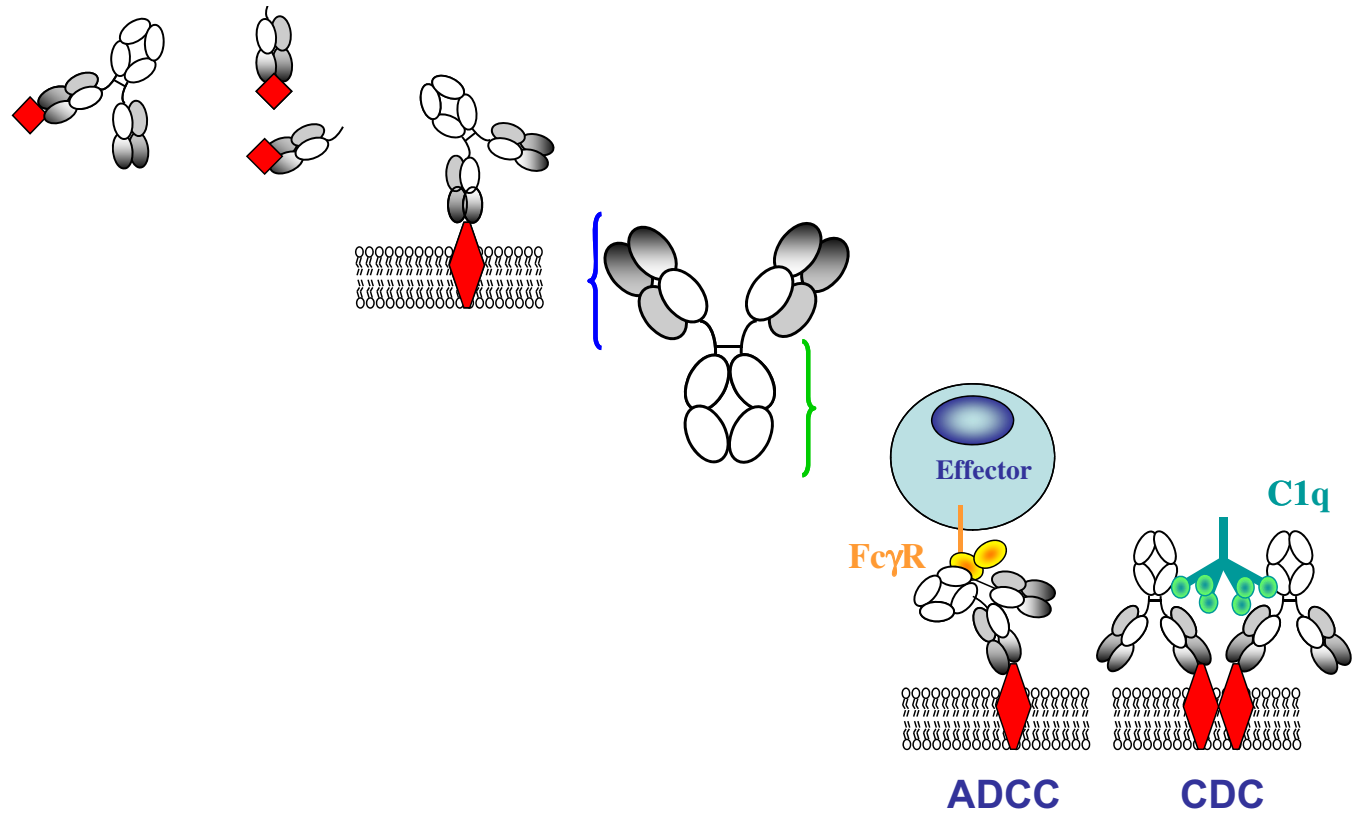

Fig. 2. For neutralising or agonist MoAb, the Fab portion is the decisive factor, particularly its affinity for the target antigen (shown in red), whether of a circulating or membrane nature. As regards cytolytic antibodies, the Fc portion also plays an important role since it is responsible for recruiting immune effectors: complement $\mathrm{C} 1 \mathrm{q}$ fraction (complement dependent cytotoxicity, or CDC) or effector cells carrying the FcyRIIIA/CD16 receptor (antibody dependent cellular cytotoxicity or $\mathrm{ADCC})$.

sclerosis, Alzheimer's disease) and metabolic disorders (osteoporosis, hypercholesterolaemia, and diabetes).

For manufacturers, the economic value of developing a MoAb is based on the following points:

- therapeutic efficacy is sometimes spectacular. An action on key players in the pathophysiology of disease is possible;

- knowledge of potential targets is rapidly growing. The number of antigens that can be targeted by MoAb is potentially infinite; the only limitation is that the antigen must be accessible to the antibody, i.e. extracellular;

- the attrition of MoAb during clinical development is lower than for "conventional" drugs (small molecules), namely because they cause fewer drug interactions and fewer undesirable effects in the short term;

- as MoAb have mainly been developed in rare diseases or indications, where needs are high, the Transparency Committee of HAS has mainly perceived them as representing advances in medicine (awarded $>$ level V improvement in the medical service rendered [amélioration du service médical rendu or ASMR]).

However, this is offset by the following elements:

- the immunogenicity of MoAb may cause the patient to produce anti-MoAb antibodies, responsible for secondary loss of response and/or undesirable effects;

- occasionally dramatic long-term immunological undesirable effects (progressive multifocal leukoencephalopathy or PML) have been observed;
- although the target antigen is perfectly defined, there is often inadequate knowledge of the mechanism of action of the MoAb in humans. The specificity of the target does not necessarily ensure "targeted" therapy for the disease;

- for a MoAb which is active in several disorders, development should be specific to the disorder studied;

- certain effector mechanisms may depend on the patient's potential for immune system response, which may be impaired by the disorder and concomitant medication (see below).

\subsection{Recommendations issued by the regulatory bodies}

A number of guidelines concerning the development of MoAb have been issued by the Food and Drug Administration (FDA), the European Medicines Agency (EMA) and the ex-Afssaps (which became the French National Agency for the Safety of Medicinal and Health Products or ANSM, in 2012). Guidelines on the quality of biopharmaceuticals, including MoAb, were published in 2008 by the EMA. ${ }^{[12]}$ A number of guidelines exist in the field of preclinical safety studies of biopharmaceuticals. There are also guidelines on the study of biopharmaceutical immunogenicity ${ }^{[13]}$ and, more specifically, that of MoAb. ${ }^{[14]}$ Further to the Tegenero incident (acute multiple organ failure occurring in healthy volunteers having received an agonist anti-CD28 MoAb), ${ }^{[15]}$ the ex-Afssaps (at present 
ANSM $)^{[16]}$ followed by the EMA ${ }^{[17]}$ published guidelines to mitigate the risks of undesirable effects during the first administration of an innovative medicinal product to humans. Recent guidelines relate to the development of biosimilars, including MoAb. ${ }^{[18]}$ However, there are no guidelines on the clinical development of MoAb.

\subsection{Limitations of animal models}

The use of animal models during the development of MoAb is difficult due to the between-species differences of the "partners" of the MoAb, i.e. target antigen and immune effectors (including Fc $\gamma \mathrm{R}$ receptors). ${ }^{[19]}$ MoAb do not usually recognise target antigens in the mouse. These models therefore require the use of a surrogate MoAb, which also binds to receptor homologues in the animal or the use of transgenic animals expressing the human target antigen. A final option involves the use of a MoAb which recognises a surrogate antigen, different from that targeted in humans, but giving rise to similar effects. Furthermore, since the antibody studied is chimeric, humanized or human, it leads to rapid immunization of the animal used as model. In view of these limitations, human cell models play a very important role in preclinical development.

\subsection{Cytolytic antibodies (oncology)}

The mode of action of MoAb in humans is still unclear. However, ADCC has been demonstrated for certain antibodies, particularly by pharmacogenetic studies. The number and efficacy of patients' immune effectors will therefore influence their clinical response. This may have a number of consequences, mainly in oncology, where MoAb are often studied as second- or third-line therapy, especially in the early phases of clinical development. Patients are at an advanced stage of disease and will have already received treatment with cytotoxic chemotherapy which may have affected their cell immunity. The MoAb should thus be studied in patients who are not at a too advanced stage of disease, and it is advisable to explore cell effectors more systematically (particularly NK cells) by counting lymphocyte sub-populations and also by studying their function ex vivo in exploratory studies. It is currently difficult to predict response capacity based on immunological assessments, due to the lack of standard tests. However, documenting the immune status of patients included in the study will help to understand the sources of interindividual variability in terms of response in the early phases. This approach may also prevent from underestimating the efficacy of a MoAb which may have been tested in patients with inactive immune effectors due to their illness, previous or concomitant medication, or a specific $\mathrm{Fc}$ receptor genotype. It would also be worthwhile measuring tumour invasion by NK cells as this is supposedly a good biomarker for response to the MoAb. Study of the FcyRIIIA receptor genotype in patients included in the study is also warranted.

\subsection{Immunogenicity}

The risk of developing immunization against the MoAb appears to be non-existent in cancer. However, in autoimmune diseases, humanization has not resolved the problems of immunogenicity since $28 \%$ of patients developed immunization against adalimumab, a fully human MoAb, after 3 years of treatment, $2 / 3$ of whom during the first 28 weeks. ${ }^{[3]}$ It is essential to explore the clinical consequences of this immunization. This mainly involves an important decrease in MoAb concentrations associated with loss of response, but also severe undesirable effects related to immunization. ${ }^{[3]}$ A number of in vitro tests to predict the immunogenicity of new biopharmaceuticals have been proposed, but their performance is still unclear.

\subsection{Early phases (I and II)}

From the first administration to humans, the quality of the clinical batches is paramount, mainly because aggregates are a key factor for immunogenicity. The mechanism of action of MoAb in humans in vivo is difficult to study, and there are many pitfalls. The risk of immunological reactions following the first few doses may correspond to:

- cytokine release syndrome (common);

- anaphylactic reactions (IgE-mediated, rare);

- target-mediated adverse side effects, related to the presence of the target antigen in healthy tissue (e.g. anti-EGFR MoAb and skin rash).

The guidelines recommend calculating the first dose on the basis of the minimum anticipated biological effect level (MABEL) rather than the no observed adverse effect level (NOAEL). ${ }^{[17]}$

However:

- this is a highly conservative approach which considerably increases the duration of trials in the early phases. It, moreover, gives rise to a risk of loss of chance for patients receiving low doses;

- it above all seems necessary for MoAb with a new mechanism of action and a membrane target, for which acute immunological undesirable effects cannot be ruled out;

- it requires an immunological expertise so that the results observed in preclinical animal or cells models can be extrapolated to humans;

- as a precautionary measure, during the very first administration (and sometimes at each dose level), it may be advisable for only a small number of subjects/patients to be exposed to the product at a time ( 1 or 2 "sentinel" subjects) before the entire cohort is exposed.

There is also the question as to the population enrolled for first administration: should healthy subjects or patients be studied? This choice is based on ethical (risk/benefit ratio) and scientific considerations, particularly the presence of the target, and the expected 


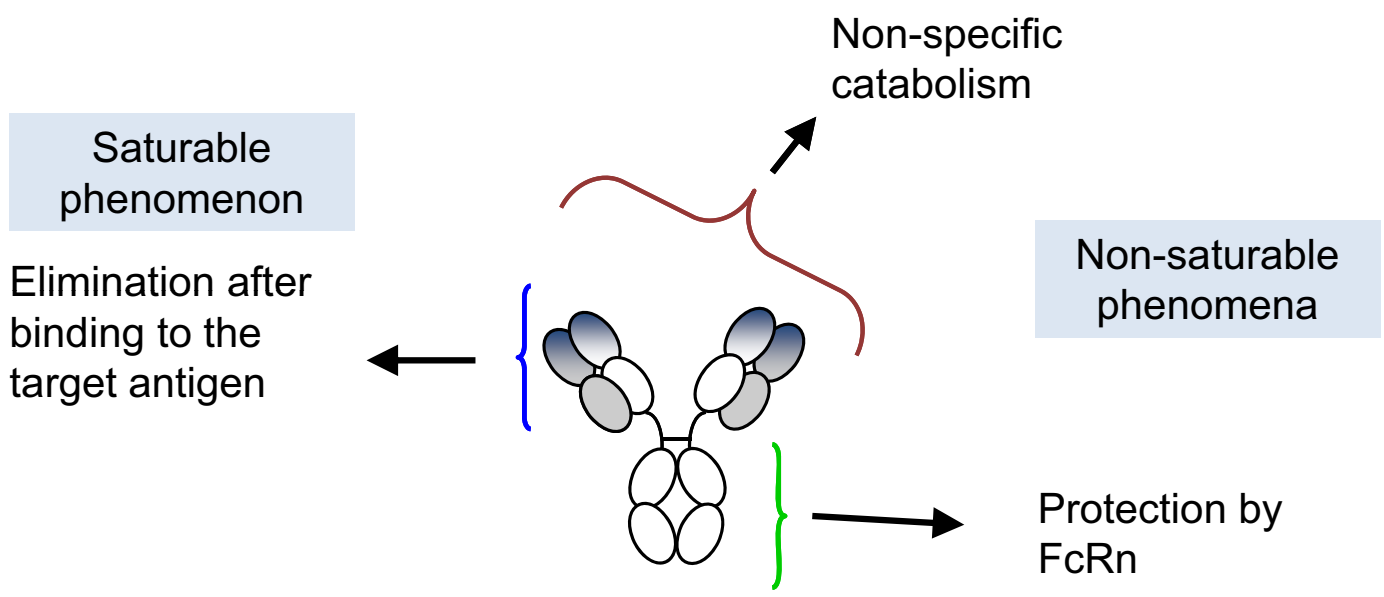

Fig. 3. MoAb are eliminated by non-specific catabolism, and are partly protected by the neonatal Fc receptor or FcRn. These two phenomena are not saturable. They are also eliminated after binding to the target antigen. Due to the limited quantity of the latter, by nature, this mode of MoAb elimination is saturable.

clinical and laboratory test results, but it may profoundly modify the results. Other questions remain open:

- should doses adjusted to weight or fixed doses be selected? The latter approach seems more relevant;

- should first administration be intravenous (so as to be able to stop the infusion in the event of an immediate reaction) or directly subcutaneous, if the latter route is chosen for development?

During phase II, a well-reasoned choice of dose is difficult for two reasons:

- good biomarkers are essential, namely "immuno-monitoring", but there is often a cascade of events between the measured biomarkers and the therapeutic effect. This "disconnection", at least temporal, between the therapeutic effect and biomarkers makes it difficult to interpret pharmacokinetic-pharmacodynamic (PK-PD) studies;

- the concentration-toxicity relationship is unclear. Toxicity is mainly dependent on the target, unlike "conventional" drugs: this involves an exaggeration of the pharmacodynamic activity, or long-term "accumulation" of the effect, e.g. in the form of immunosuppression.

Analysis of the MoAb currently on the market shows that doses are sometimes selected empirically. This reflects the difficulty of the clinical development of MoAb, particularly during the early phases, which are probably insufficiently codified.

\subsection{Pharmacokinetics}

After subcutaneous or intramuscular administration, the absorption of MoAb is very slow, reaching a peak after approximately one week. The mechanisms for elimination of MoAb are very different from "conventional" drugs. On the one hand, they undergo non-specific catabolism, IgG being degraded like other circulating proteins by vascular endothelial cells, which is not a saturable phenomenon. On the other hand, MoAb are eliminated after binding to their target antigen, by internalization when the target is a membrane receptor or by forming immune complexes for circulating targets. ${ }^{[20]}$ Because the amount of target antigen is, by nature, limited, this mode of MoAb elimination is saturable. The third mechanism involved in the elimination of MoAb is their protection against degradation by to a specific receptor, neonatal Fc receptor or FcRn. ${ }^{[21]}$ This protection explains their long half-life of approximately 3 weeks (figure 3 ). When circulating proteins are passively uptaken by vascular endothelial cells, the endosomes gradually become acidic, and the proteins are degraded in lysosomes. FcRn, present in endocytotic vesicles, binds to the Fc portion of the antibodies and diverts them from this degradation pathway towards the apical cell membrane. This phenomenon is not saturable at therapeutic MoAb concentrations. In terms of pharmacokinetic modelling, the elimination of MoAb is usually therefore non-linear, and should be described by both non-saturable and saturable phenomena. FcRn, present in numerous types of tissue, is also responsible for transcytosis and, hence, tissue distribution of antibodies. The antibodies are not therefore confined within the systemic circulation. FcRn is notably responsible for transplacental passage of maternal antibodies (natural antibodies or MoAb) at the end of pregnancy, and expulsion of antibodies from the central nervous system (which explains the low passage of intravenously injected MoAb). ${ }^{[20,21]}$

Certain sources of interindividual variability of MoAb pharmacokinetics differ from "conventional" drugs. Since binding to the target antigen gives rise to the elimination of the antibody, the "antigen mass" has an impact on pharmacokinetics. ${ }^{[22]}$ The quantity of target antigen varies between patients, whether in the context of tumour disease or inflammatory disorders. Disease activity will 


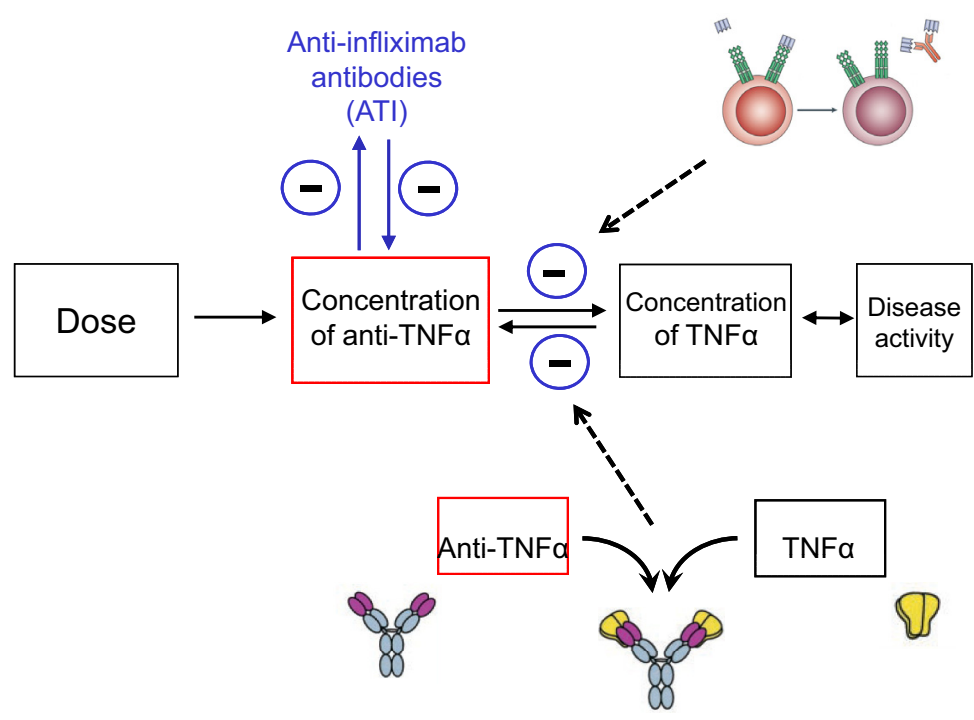

Fig. 4. The elimination of MoAb after binding to their target antigen means that the quantity of target antigen will have an impact on pharmacokinetics, as in the case of anti-TNF $\alpha$ MoAb. The risk of immunisation is higher the lower the MoAb concentrations. When immunisation occurs, anti-MoAb antibodies reduce MoAb concentrations. MoAb, the target antigen and the induced antibodies are therefore inter-related. Notably, if there is high disease activity, with high target antigen concentrations, MoAb concentrations will be reduced, and the risk of immunisation and, hence, loss of response will increase.

therefore influence MoAb clearance (figure 4). This two-way relationship should ideally be described by a target-mediated drug disposition (TMDD) model, which describes both the pharmacokinetic profile and PK-PD relationship. ${ }^{[23]}$

As discussed above, patient immunization will be responsible for reduced MoAb concentrations. This immunisation is in itself dependent on the concentrations of the therapeutic antibody since, for example, the risk of developing anti-infliximab antibodies has been shown to be higher the lower the infliximab concentrations. ${ }^{[24,25]}$ The activity of inflammatory disease could therefore be indirectly responsible for the onset of induced antibodies, and therefore secondary loss of response (figure 4). Furthermore, anti-MoAb antibodies may be present before first administration of the biopharmaceutical, as shown for anti- $\alpha$ GAL IgE directed against cetuximab ${ }^{[26]}$ or when the Fc portion of MoAb has been modified. ${ }^{[27]}$

\section{Evaluation by the agencies and long-term monitoring of safety}

\subsection{Evaluation by the ex-Afssaps Marketing Authorisation Committee}

Marketing authorisations for MoAb, as drugs derived from biotechnological processes, are granted in the context of a centralised European procedure after evaluation by the EMA, with two countries acting as rapporteurs and the others, recipients. Insofar as the expertise in these biotechnological products should be associated with an expertise in the treated disorder, it does not appear necessary to set in place a specialized evaluation group alongside the marketing authorisation committee which would be exclusively responsible for evaluation of the MoAb.

\subsection{Evaluation by the Transparency Committee of the French National Authority for Health}

There are numerous indications for MoAb. Since 1999, the French National Authority for Health (HAS) Transparency Committee has analysed with a view to their reimbursement 22 proprietary medicinal products in 45 indications: 12 with 1 indication, 5 with 2 indications, 1 with 4 indications, and 4 with 5 indications (one indication being able to obtain several ASMR levels, e.g. according to the line of treatment). The medical service rendered (SMR) was perceived as important on 42 occasions, and moderate or low on 4 occasions. The ASMR was perceived as major (level I) to moderate (level III) on 23 occasions, and minor (IV) on 11 occasions. In 11 cases, a MoAb was not perceived as representing progress in relation to the existing treatment (ASMR V) and on one occasion the ASMR could not be assessed. The evaluation of $\mathrm{MoAb}$ is not different from other drugs (the same review process is used), but the treatment situations (serious and/or rare diseases) have, until now, been unusual. 


\subsection{Long-term safety studies}

Safety studies are only some of the postmarketing studies requested by the HAS Transparency Committee. Out of a total of 166 studies requested, 32 studies concerned $15 \mathrm{MoAb}$ proprietary medicinal products: 15 studies on the prescribing/usage conditions, 9 studies on the benefit to patients in terms of morbidity/ mortality, 2 studies on the impact on the healthcare system, and 6 safety studies. As regards MoAb, long-term safety studies appear to be justified due to:

- the serious or rare nature of the disorder;

- the novel nature of the target antigen, the clinical consequences of its interaction with the MoAb being unclear;

- the incomplete evaluation of the dosing regimen (dose and treatment duration);

- the disconnection between short-term and long-term toxicity, together with the absence of data on long-term toxicity at the time of the evaluation for marketing and first inclusion on the list of reimbursed products.

The Round Table discussed the possibility of adapting the postmarketing risk management plans (RMP) requested by the EMA (or specifically by the ex-Afssaps [at present ANSM]) more closely to MoAb, since certain undesirable effects are expected due to their relatively specific mode of action (perfectly defined target antigen) and the knowledge of MoAb format (cytolysis expected for IgG1 for instance). However, there are still unexpected effects, and certain expected effects are not observed. The development of MoAb should clearly be accompanied by an immunological expertise so as to help prevent all of these effects; however, on the opposite, the use of MoAb has improved the immunological knowledge. Therefore, undesirable effects cannot yet be fully predicted, and caution is still necessary.

\subsection{Observatories on medicinal products, medical devices and therapeutic innovation}

Observatories on medicinal products, medical devices and therapeutic innovation (OMEDITs) allow the analysis of prescribing conditions for MoAb in "real-life" situations, to conduct cost/efficacy studies, and to respond to requests made by the Transparency Committee as part of the postmarketing surveillance of MoAb. The observatory dedicated to cancer in the Brittany and Pays de la Loire regions is associated with the two OMEDITs of these regions. This observatory, for instance, carried out an analysis of the costs incurred by the treatment of 730 patients with metastatic colorectal cancer in 38 establishments for one line of treatment over a one-year follow-up: the median cost per patient was $€ 7,500$ for chemotherapy alone versus $€ 24,500$ for chemotherapy in combination with a MoAb. The "drug" item was higher for MoAb although MoAb had a lower "toxicity" item. ${ }^{[28]}$ Another study conducted by this observatory concerned the actual conditions of use of a combination of bevacizumab+chemotherapy in metastatic colorectal cancer among 2,050 patients, and observed a higher mean age, risk of toxicity and risk of death relative to the data in the literature. ${ }^{[29,30]}$

Postmarketing studies are essential because they provide information on the population actually treated, and on the risk/benefit ratio in this population.

\subsection{Role of scientific societies}

The HAS issues guidelines on the prescribing of drugs. It aims to inform public decision-makers, and the texts may therefore be out of step with those issued by scientific societies, which tend to focus more on the state of the art for prescribing physicians. It is important that opinions are devoid of any conflicts of interest as far as possible. The themes of the guidelines are chosen by the HAS college according to public health priorities and on the request of the minister, scientific societies, french health insurance, patient associations, and other professional bodies. Drafting is coordinated by a technical committee supported by multidisciplinary working groups and external reviewers. The final version is validated by the HAS college before it is issued. This procedure may therefore take longer (approximately 18 months) than that of guidelines from other sources.

Postmarketing surveillance may also be carried out in the context of registries, following up cohorts of patients treated with MoAb, set in place by scientific societies, often in partnership with the agencies and/or manufacturers; however, the latter are not involved in designing or carrying out follow-up. The example of rheumatology was discussed during the Round Table as 20 European registries have been identified for this discipline, including 4 in France: "RATIO", an in-depth pharmacovigilance programme for the collection of opportunistic infections and lymphomas in patients treated with anti-TNF; "AIR", follow-up of patients treated with rituximab for autoimmune disease; "ORA", follow-up of patients treated with abatacept for rheumatoid arthritis, and, lastly, "REGATE", follow-up of patients treated with tocilizumab for rheumatoid arthritis. ${ }^{[31]}$ Although these registries enable a great deal of information to be collected, better coordination between the different protagonists (scientific societies, pharmaceutical industry, and agencies) would be desirable.

\section{Conclusion}

MoAb, which can be designed to act on a potentially infinite number of targets and functions, are innovative drugs: their novel modes of action is a challenge for clinical pharmacology. The Round Table did not identify any characteristic justifying a specific 
methodology for clinical development and evaluation. However, the analysis identified a set of distinctive features of MoAb compared with "conventional" drugs:

- the early phases of clinical development give rise to specific problems, namely regarding the choice of the first dose to be tested, and the type of subjects to be included;

- the pharmacokinetic profile of MoAb is very different from that of "conventional" drugs;

- toxicity is mainly target-dependent, with exaggeration of response related to their pharmacodynamic activity, e.g. short-term or long-term immunosuppression;

- the PK-PD relationship is difficult to study because the therapeutic effect may be related to a cascade of events, and apparently disconnected from accessible biomarkers;

- the therapeutic effect of cytolytic MoAb brings into play the patient's immune system, which varies due to genetic, physiological and pathological reasons.

Although the evaluation of MoAb by the health authorities is not different from that of "conventional" drugs, the distinctive feature of MoAb stems from a combination of several factors: the disorders treated are serious and/or rare; a new target antigen is often involved, the dosing regimen (dose and treatment duration) are not fully assessed during clinical development, and there is disconnection between short-term and long-term toxicity, the latter being inadequately described at the time of marketing.

\section{Participants.}

Martine Bagot (UniversitéParis Diderot Sorbonne, AP-HPHôpital Saint-Louis, Inserm U976), Isabelle Boucot (GSK), Anne d'Andon (Haute Autorité de Santé), Françoise Grudé (Observatoire dédié au Cancer Bretagne Pays de la Loire, ICO Paul Papin Angers), Thierry Lecomte (Université François Rabelais Tours, CHRU de Tours, CNRS UMR 7292), Soyi Liu-Leage (Lilly), Daniel Olive (Aix Marseille Université, Institut Paoli Calmettes, Inserm), Pascal Piedbois (Astra Zeneca), Martine Pigeon (CNAMTS), Jean-Louis Pinquier (Sanofi R\&D), Jean-François Prost (LFB), Gilles Thibault (Université François Rabelais Tours, CHRU de Tours, CNRS UMR 7292), Christian Vitzling (Novartis).

Conflicts of interest. Gilles Paintaud leads a research team which receives funding for the Laboratoire français de fractionnement et de biotechnologie (LFB) and from Pierre Fabre Laboratory, Novartis, Roche Pharma, Pfizer, Janssen and Chugai. Marine Diviné is employee of Amgen France. Philippe Lechat has no conflict of interest to declare.

\footnotetext{
Abbreviations. ADCC: antibody dependent cellular cytotoxicity; (ex-)Afssaps : ex-Agence française de sécurité sanitaire des produits de santé (at present french National Agency for the Safety of Medicinal and health Products [ANSM]; anti-RANK-ligand: antireceptor activator of nuclear factor-kappaB ligand antibody; antiTNF $\alpha$ : anti-tumor necrosis factor- $\alpha$; anti-VEGF : anti-vascular
}

endothelial growth factor; ASMR : amélioration du service médical rendu (improvement of medical service rendered); CDC: complement dependent toxicity; CDR: complementarity determining region; EMA : European Medicines Agency; FDA: Food and Drug Administration ; HAS: Haute autorité de santé (french National Authority for Health); HER: epidermal factor receptor family; IgG : immunoglobulin G; MABEL: minimum anticipated biological effect level ; MoAb: monoclonal antibodies; NK: natural killer; NOAEL: no observed adverse effect level ; PML : progressive multifocal leukoencephalopathy; OMEDITs: Observatories on Medicinal Products, Medical Devices and Therapeutic Innovation; PK-PD: pharmacokinetic-pharmacodynamic; RMP: postmarketing risk management plans; SMR: service médical rendu (medical service rendered); TMDD: target-mediated drug disposition.

\section{References}

1. Paintaud G, Tonelli D, Postaire E, et al. Biotherapies: are they just like any other drugs? Therapie 2007; 62: 235-39

2. Paintaud G, Lejarre F, Ternant D, et al. Les anticorps monoclonaux : une avancée thérapeutique récente et majeure. Therapie 2009; 64: 1-7

3. Bartelds GM, Krieckaert CL, Nurmohamed MT, et al. Development of antidrug antibodies against adalimumab and association with disease activity and treatment failure during long-term follow-up. JAMA 2011; 305: 1460-8

4. Mitoma $\mathrm{H}$, Horiuchi $\mathrm{T}$, Tsukamoto $\mathrm{H}$, et al. Mechanisms for cytotoxic effects of anti-tumor necrosis factor agents on transmembrane tumor necrosis factor alpha-expressing cells: comparison among infliximab, etanercept, and adalimumab. Arthritis Rheum 2008; 58: 1248-57

5. Congy-Jolivet N, Probst A, Watier H, et al. Recombinant therapeutic monoclonal antibodies: mechanisms of action in relation to structural and functional duality. Crit Rev Oncol Hematol 2007; 64: 226-33

6. Lejeune $\mathrm{J}$, Thibault $\mathrm{G}$, Cartron $\mathrm{G}$, et al. Rôle des récepteurs à la portion $\mathrm{Fc}$ des IgG (FcyRs) dans l'activité des anticorps thérapeutiques. Bull Cancer 2010; 97: 511-22

7. Cartron G, Dacheux L, Salles G, et al. Therapeutic activity of humanized anti-CD20 monoclonal antibody and polymorphism in $\mathrm{IgG} \mathrm{Fc}$ receptor FcgammaRIIIa gene. Blood 2002; 99: 754-8

8. Musolino A, Naldi N, Bortesi B, et al. Immunoglobulin G fragment C receptor polymorphisms and clinical efficacy of trastuzumab-based therapy in patients with HER-2/neu-positive metastatic breast cancer. J Clin Oncol 2008; 26: 1789-96

9. Bibeau F, Lopez-Crapez E, Di Fiore F, et al. Impact of Fc $\gamma$ RIIa-Fc $\gamma$ RIIIa polymorphisms and KRAS mutations on the clinical outcome of patients with metastatic colorectal cancer treated with cetuximab plus irinotecan. J Clin Oncol 2009; 27: 1122-9

10. Béliard R, Waegemans T, Notelet D, et al. A human anti-D monoclonal antibody selected for enhanced Fc $\gamma$ RIII engagement clears $\mathrm{RhD}^{+}$autologous red cells in human volunteers as efficiently as polyclonal anti-D antibodies. Br J Haematol 2008; 141: 109-19

11. De Romeuf C, Dutertre CA, Le Garff-Tavernier M, et al. Chronic lymphocytic leukaemia cells are efficiently killed by an anti-CD20 monoclonal antibody selected for improved engagement of Fc $\gamma$ RIIIA/CD16. Br J Haematol 2008; 140: 635-43

12. EMA. Guideline on development, production, characterization and specifications for monoclonal antibodies and related products. $2008 \mathrm{CHMP} / \mathrm{BWP} /$ 157653/2007. http://www.ema.europa.eu 
13. EMA. Guideline on immunogenicity assessment of biotechnology-derived therapeutic proteins. 2007 EMEA/CHMP/BMWP/14327/2006. http:// www.ema.europa.eu

14. EMA. Guideline on immunogenicity assessment of monoclonal antibodies intended for in vivo clinical use. 2010 EMEA/CHMP/BMWP/86289/2010. http://www.ema.europa.eu

15. Suntharalingam G, Perry MR, Ward S, et al. Cytokine storm in a phase 1 trial of the anti-CD28 monoclonal antibody TGN1412. N Engl J Med 2006; 355: $1018-28$

16. Afssaps. Essais cliniques de première administration à l'Homme, en dose unique d'un médicament expérimental (nouvelle substance active) : choix de la première dose, de la progression de dose et protocole d'administration aux volontaires. 2006. http://ansm.sante.fr/S-informer/Presse-Communiques-Points-presse/Premiere-administration-d-un-medicament-experimental-chez-I-homme-I-Afssaps-diffuse-des-recommandations-sur-lesessais-cliniques-de-phase-1/(language)/fre-FR

17. EMA. Guideline on requirements for first-in-man clinical trials for potential high-risk medicinal products. 2007 CHMP/SWP/28367/2007. http:// www.ema.europa.eu

18. EMA. Guideline on similar biological medicinal products containing monoclonal antibodies. 2010 EMEA/CHMP/BMWP/403543/2010. http:// www.ema.europa.eu

19. Loisel S, Ohresser M, Pallardy M, et al. Relevance, advantages and limitations of animal models used in the development of monoclonal antibodies for cancer treatment. Crit Rev Oncol Hematol 2007; 62: 34-42

20. Paintaud G. Pharmacocinétique des anticorps monoclonaux. Med Sci (Paris) 2009; 25: 1057-62

21. Magdelaine-Beuzelin C, Ohresser M, Watier H. FcRn, un récepteur d'IgG aux multiples facettes. Med Sci (Paris) 2009; 25: 1053-6

22. Ternant D, Paintaud G. Pharmacokinetics and concentration-effect relationships of therapeutic monoclonal antibodies and fusion proteins. Expert Opin Biol Ther 2005; 5: S37-S47

23. Yan X, Mager DE, Krzyzanski W. Selection between Michaelis-Menten and target-mediated drug disposition pharmacokinetic models. J Pharmacokinet Pharmacodyn 2009; 37: 25-47
24. Bendtzen K, Geborek P, Svenson M, et al. Individualized monitoring of drug bioavailability and immunogenicity in rheumatoid arthritis patients treated with the tumor necrosis factor inhibitor infliximab. Arthritis Rheum 2006; 54: 3782-9

25. Ducourau E, Mulleman D, Paintaud G, et al. Antibodies toward infliximab are associated with low infliximab concentration at treatment initiation and poor infliximab maintenance in rheumatic diseases. Arthritis Res Ther 2011; 13: R105

26. Chung $\mathrm{CH}$, Mirakhur B, Chan E, et al. Cetuximab-induced anaphylaxis and IgE specific for galactose-alpha-1,3-galactose. N Engl J Med 2008; 358: 1109-17

27. Zheng Y, Scheerens H, Davis JC Jr, et al. Translational pharmacokinetics and pharmacodynamics of an FcRn-variant anti-CD4 monoclonal antibody from preclinical model to phase I study. Clin Pharmacol Ther 2011; 89: 283-90

28. Grudé $\mathrm{F}$, Roué $\mathrm{V}$, Beauchamp $\mathrm{C}$, et al. Real cost linked to the introduction of targeted therapies (bevacizumab, cetuximab) and conventional chemotherapies in the treatment of metastatic colorectal cancer. Gastrointestinal Cancers Symposium, San Francisco 2009, A330

29. Hurwitz HI, Fehrenbacher L, Hainsworth JD, et al. Bevacizumab in combination with fluorouracil and leucovorin: an active regimen for first-line metastatic colorectal cancer. J Clin Oncol 2005; 23: 3502-8

30. Metges JP, Grudé F, Faroux R, et al. FOLFIRI bevacizumab in unresectable metastatic colorectal cancer in the true life, feasible in elderly patients: results of the cohort from OMIT Bretagne - Pays de Loire. Gastrointestinal Cancers Symposium, San Francisco, A398

31. Mariette X, Gottenberg JE, Ravaud $\mathrm{P}$, et al. Registries in rheumatoid arthritis and autoimmune diseases: data from the French registries. Rheumatology 2011; 50: 222-9

Correspondence and offprints: Gilles Paintaud, Pharmacology-Toxicology Laboratory and CNRS UMR 7292, CHRU of Tours, 2 boulevard Tonnellé, F-37044 Tours Cedex 9, France.

Email: paintaud@med.univ-tours.fr 